Man and Nature

L'homme et la nature

\title{
Les Moralistes des Lumières et la Révolution
}

\section{Henri Mydlarski}

Volume 6, 1987

URI : https://id.erudit.org/iderudit/1011878ar

DOI : https://doi.org/10.7202/1011878ar

Aller au sommaire du numéro

Éditeur(s)

Canadian Society for Eighteenth-Century Studies / Société canadienne d'étude du dix-huitième siècle

ISSN

0824-3298 (imprimé)

1927-8810 (numérique)

Découvrir la revue

Citer cet article

Mydlarski, H. (1987). Les Moralistes des Lumières et la Révolution. Man and Nature / L'homme et la nature, 6, 181-191. https://doi.org/10.7202/1011878ar

Copyright (c) Canadian Society for Eighteenth-Century Studies / Sociéte canadienne d'étude du dix-huitième siècle, 1987
Ce document est protégé par la loi sur le droit d'auteur. L'utilisation des services d'Érudit (y compris la reproduction) est assujettie à sa politique d'utilisation que vous pouvez consulter en ligne.

https://apropos.erudit.org/fr/usagers/politique-dutilisation/ 


\section{LES MORALISTES DES LUMIÈRES ET LA RÉVOLUTION}

"La clarté orne les pensées profondes,"1 écrit Vauvenargues dans ses Réflexions et Maximes. Remarque bien anodine en apparence. Bien innocente aussi dans son énoncé. Remarque que rend pourtant plus actuelle que jamais le sabir de tant d'études modernes et qui, dans son laconisme, suffit à sanctionner avant son heure l'appellation de "Lumières" pour le XVIIIle siècle et à investir la production de tout discours critique (si l'on ne veut pas être complètement dupe des autres et de ... soi-même) d'un double principe d'intelligibilité et de cohérence. Pour me soustraire à l'obscur que produit toujours le galvaudage des termes, et parce qu'il me semble, comme du reste à Voltaire qui voyait en Vauvenargues un génie aussi rare que Pascal, qu' 'il n'y aurait point d'erreurs qui ne périssent d'elles-mêmes, rendues clairement, ${ }^{\prime 2}$ je précise donc que je prends moraliste dans son sens traditionnel, c'est-à-dire dans l'acception qui recouvre des noms tels que Pascal, La Rochefoucauld, La Bruyère ... pour le XVIIe siècle et Duclos, Vauvenargues, Toussaint, Sénac de Meilhan, Chamfort, Rivarol, Joubert ... pour le XVIIIe.

Le champ de mon propos étant ainsi délimité, les moralistes formant, par ailleurs, un genre particulièrement français de la République des lettres, ${ }^{3}$ un peu comme si "la France [était] un pays de moralistes", ${ }^{4}$ ainsi que l'estime Siegfried, j'en suis venu naturellement à me demander quelle part, directe ou indirecte, ils avaient pu avoir à la Révolution. Car il serait naif de croire, comme le laisse croire la définition du Robert, 
qu'ils ne sont que des écrivains qui observent et peignent les moeurs, des auteurs de réflexions sur les habitudes des hommes, et, en général, sur la condition et la nature humaines. Personne n'est seul de son espèce ni de son époque. Et nos prétendus universalistes ne sont pas plus imperméables au politique sanguin de la théologie par exemple qu'au théologique éthéré de la politique. A une époque où le domaine de la science morale de l'individu et, a fortiori, de la société se reçoit des mains de l'Eglise et de sa mission apostolique pour informer (au sens thomiste de donner une forme) chrétiennement la nation, les moralistes des Lumières ont beau regarder la vie en face sans puérilité ni hypocrisie, ils ont aussi leurs illusions, leurs rêves, leurs fantasmes, leurs psychoses, leurs espoirs, leurs estimations valorielles ... A leurs façons respectives, leurs personnalités bouleverseront aussi le monde. Davantage, à s'élever -- ou mieux: à faire croire qu'ils s'élèvent jusqu'au désintéressement intellectuel; à souvent s'enfermer, pour laisser penser qu'ils se tiennent au-dessus de la mêlée, dans un langage apophtegmatique qui, par sa nature oraculaire, interdit la discussion, ils portent à l'Ancien Régime des coups d'autant plus subversifs qu'ils semblent partir d'une position d'inviolabilité "sacerdotale". Ils substituent à la Révélation, qui dévoile le mystère de Dieu pour mieux investir de son autorité le Trône et l'Autel, la Raison, qui énonce la vérité profane de l'homme pour mieux le rendre à sa vocation laïque et naturelle: "L'homme, écrit par exemple Vauvenargues, est maintenant en disgrâce chez tous ceux qui pensent, et c'est à qui le chargera de plus de vices; mais peut-être est-il sur le point de se relever, et de se faire restituer toutes ses vertus". ${ }^{5}$ Sur quoi le critique-économiste que fut au milieu du siècle dernier Henri Baudrillart déclare: "Oui, Vauvenargues, vous l'avez dit: tous ceux qui vont venir n'y manqueront pas. Ils vont restituer à l'homme ses vertus et bien au delà: ...injures qu'elle [la nature humaine] reçoit depuis dix-huit cents ans de tous les côtés vont être bien réparées; et ... cette reine déchue et réduite en servitude, une fois replacée sur son trône, n'aura plus désormais que des flatteurs qui la diviniseront." ${ }^{\prime 6}$ Baudrillart s'empresse même d'ajouter que le moraliste avance là "tout un système nouveau, et comme le renversement de la théorie consacrée." ${ }^{\prime 7}$ Outrance de propos sans doute, peut-être même réductionnisme facile de l'histoire des mentalités en France: ce Vauvenargues qui dénonce (entre autres) le dogme de la chute comme un outrage au simple bon sens; dont la libre réflexion critique est appelée à ruiner par ses retombées les instances politiques et religieuses qui encadrent et s'approprient l'homme, ce Vauvenargues, il n'est pas alors unique, il ne vient pas proclamer un évangile tout neuf. Compagnon de la guerre intellectuelle de son 
temps, il en est aussi le témoin. Instigateur primitif de la Révolution? Il ne saurait l'être. Paul Hazard ne montrait-il pas naguère que la crise de la conscience française, largement européenne de fond, remontait à l'avant-dernière décennie du XVIIe siècle? Et pourtant! La relève du combat est souvent plus brutale que son impulsion première, surtout quand elle est assurée par la jeunesse qui s'imagine la prendre en pleine intégrité de jugement et à la seule force de son intelligence. Comprenons bien: point n'est question ici de nier l'originalité de Vauvenargues. Plutôt, il s'agit de ne pas l'exclure outre mesure, pas plus, au demeurant, que celle des autres moralistes, de la grande laïcité des Lumières, du déjà historique mouvement des idées qui mènera à la Révolution. Car, à faire "le procès du christianisme", 8 tous préparent les esprits à la cité terrestre, nullement à la Jérusalem céleste. Et c'est encore Baudrillart qui écrit: "il ne faut pas se dissimuler que la résignation religieuse, admise comme premier principe et poussée jusqu'à l'extrême, a pour effet inévitable de supprimer les problèmes que soulèvent les intérêts sociaux, de fermer dès lors à l'esprit tout un ordre d'idées grandes et fécondes". ${ }^{9}$ Mais, justement, c'est en récusant presque point sur point l'instruction pastorale de l'Eglise post-tridentine, qui continue à avoir force de loi, que les moralistes approfondiront le champ de la conscience politique et feront figure $d$ 'authentiques champions de la Parole nouvelle dès la première moitié du XVIIIe siècle. Les Réflexions et maximes de Vauvenargues, les Moeurs de Toussaint et les Considérations de Duclos datent en effet de 1746, 1748 et 1750 respectivement. Avec ces trois ouvrages, les premiers où soit avancé un projet de morale naturaliste, où le discours social s'affranchisse de son lest religieux, où enfin la collectivité soit comme mise au défi d'évacuer de son comportement séculaire le rituel ecclésiastique, se ramasse tout l'humanisme diffus du XVIIIe siècle, se font jour pour les hommes toute la dignité et tout le bonheur qu'ils méconnaissent en eux. "La vertu et le plaisir sont nés avec la nature, écrit Vauvenargues qui ajoute sur un ton ironique particulièrement humiliant pour les Ecritures et la Tradition: qu'est-ce que le reste?"11 Toussaint, de son côté, avant de faire comprendre qu'il ne saurait y avoir de liturgie ni de cérémonies qui ne conduisent au crétinisme, observe que la Nature est une bonne mère, qu'elle n'a pas mis entre les individus l'inégalité qu'on lui prête, et déclare: "Pour l'homme sensé, bien-loin d'imaginer que la vivacité de sa passion, les oppositions même qu'il rencontre, et les difficultés qu'il lui faut surmonter, soient de vrais malheurs, dont il doive gémir, il les regarde au contraire comme destinés à piquer ses sens et à rehausser la saveur du plaisir. Ostez de la joüissance les désirs et les obstacles, vous en anéantissez tous les charmes". ${ }^{12}$ 
Quant à Duclos, il prend à partie, dès son premier chapitre, ceux qui supposent que "l'home n'est qu'un composé de misère et de corruption, et qu'il ne peut rien produire d'estimable". Il enchaïne: "Ce système est aussi faux que dangereux"; ${ }^{13}$ et, dans son avant-dernier chapitre, comme emporté par une ferveur déjà toute révolutionnaire, il lance le fameux: "Le meilleur des gouvernements n'est pas celui qui fait les hommes les plus heureux, mais celui qui fait le plus grand nombre d'heureux". ${ }^{14}$

Or les moralistes n'ignorent pas que c'est l'autorité qui garantit l'intégrité de l'Eglise et en assure la survivance. Toussaint ne va-t-il pas jusqu'à prétendre, avec, pour l'époque, une franchise frôlant une totale inconscience du danger censorial, qu'il serait absurde de penser que le culte extérieur ait été institué en un rapport de nécessité avec le divin? N'observe-t-il pas que les pratiques religieuses n'ont jamais servi qu'à disposer par la structuration sacramentelle un champ d'action commune et d'habitus public? À "unir, comme il le dit lui-même, les membres de la société par la profession ouverte d'une seule et même Religion?"15 Sur ce mode, il serait donc mal venu de donner créance à l'attachement que les moralistes professent pour leur foi catholique, et encore plus à leur orthodoxie. En fait, derrière leurs protestations obédientielles, ils sont générateurs "d'eutrapélie", pour parler comme Jacques Vier, c'est-à-dire d'une ironie d'autant plus accablante et d'une démarche d'autant plus faussement naïve que l'une et l'autre se soumettent davantage. "Geste inverse de celui que recommandait Pascal, bien étonné d'apprendre que l'on pût, à ce point, mésuser d'un bénitier". ${ }^{16}$ Mais aussi geste qui atteste, toujours à l'encontre de Pascal qui ne se berçait pas d'illusions, que les moralistes de cette première moitié de siècle caressent un rêve de progrès humain, de réforme politique et de justice sociale. ${ }^{17}$ De ce rêve va naître bientôt la Révolution.

Rien de plus prémonitoire à cet égard, rien qui fasse mieux sentir l'orage qui approche et qui va bouleverser le monde, que le chapitre des Considérations "sur les grands Seigneurs". Non, sans doute, le livre de Duclos ne brille guère par ses qualités proprement littéraires. Il ne donne pas non plus de l'homme ni de la société du temps qu'il prétend peindre des aperçus bien profonds. Mais son traitement de la noblesse est d'une autre facture: l'éreintement systématique qu'il lui fait subir; le mépris dont il l'enveloppe; le parasitisme dont il l'accuse; les gains honteux qu'il lui voit soustraire à la crédulité du pauvre; le faste où elle se vautre pour en imposer aux malheureux qui en font par surcroît les frais; l'ignorance et la crainte où elle tient le peuple pour mieux l'exploiter; et puis, d'une veine autrement plus explosive, l'idée qu'elle a fait son temps depuis que la France ne vit plus sous 
le régime de la féodalité médiévale; le principe, sans cesse répété, de l'égalité des hommes devant une loi commune; l'art d'escamoter par le silence, pour en réfléchir de biais le non-sens odieux, les trois ordres qui fondent la société française en la divisant; bref, tout ici, jusqu'à l'impertinence de donner à croire à une abolition plus ou moins juridique des privilèges et des droits seigneuriaux, fait mesurer le chemin parcouru depuis La Bruyère. Car il ne s'agit plus seulement de morale mais de politique. Aux élans d'indignation généreuse des Caractères se substituent dans les Considérations la froide mise à mort de la noblesse en tant que classe et la fabrication d'un pays fondé, non sur le respect de la Tradition, mais sur la vision d'un système de justice. "Ce n'est point ici une idée de république imaginaire, écrit même dans son chapitre "Sur l'éducation et sur les préjugés" Duclos qui ajoute: d'ailleurs, ces sortes d'idées sont au moins d'heureux modèles, des chimères, qui ne le sont pas totalement, et qui peuvent être réalisées jusqu'à un certain point." ${ }^{18}$ Refaire la société, voilà donc ce que se propose ce maître à penser si profondément porteur d'avenir. Il y a plus. Par son côté "fils de riches commerçants" qui ne jure que par la réalité politique de l'argent et du pouvoir, Duclos se trahit et laisse percer à son insu que la Révolution qui se prépare sera bourgeoise.

Ce virage politique, Vauvenargues aussi le prend; et sur un ton, à l'occasion, beaucoup moins pondéré que celui du futur secrétaire perpétuel de l'Académie française. Quant à son tour d'esprit, s'il peut paraitre également autre au premier abord, il n'en conduit guère moins à se pencher sur les questions à l'ordre du jour et à revendiquer une refonte de l'organisation sociale. Non, Vauvenargues ne vécut ni en indépendant ni en étranger du siècle, comme on le dit trop souvent. Et il ne demeure pas toujours guidé par une réflexion psychologique ou morale. "Que d'hommes inutiles en France! s'écrie-t-il par exemple dans un souffle déjà révolutionnaire: que de légistes, que de valets, que de religieux! que de bourgeois, qui croupissent dans l'oisiveté des villes, et privent le royaume de leur industrie et de leur travail!"19 Assurément, ne sont pas visés ici les nobles. Le marquis de Vauvenargues pourrait-il décemment déroger à sa qualité de seigneur? Pourrait-il se dérober honnêtement au rang qu'il fustigerait? Mais chacun a son "défaut" où toujours il revient, dit le poète. Que la distinction des ordres du royaume soit "une des lois fondamentales de l'Etat", ${ }^{20}$ comme il l'avance, ou encore que la noblesse soit "un héritage, comme l'or et les diamants", ${ }^{21}$ rien pourtant ne saurait empêcher le-dit "seigneur" de mettre implacablement en faillite le système même qu'il prétend sauvegarder. "Voyez ces grands, écrit-il par exemple sur un ton de protestation indignée, si somptueux dans leur train, mais d'autant plus 
pauvres en vertu, sans autorité à la cour, sans considération dans les provinces, sans réputation dans les armées, réduits à leurs flatteurs et à leurs domestiques pour clients: plusieurs jouissent dans l'opprobre de la récompense méritée par leurs pères, comme si les grandes places de l'Etat devaient être l'héritage de la vanité et de la mollesse!'”22 Eloquent (et ironique) renversement du pour au contre, discours digne des plus hauts moments de la tribune révolutionnaire, qui ne fait pas que réduire à néant la thèse contraire qu'il s'emploie ailleurs à défendre mais qui réfracte avec acuité le malaise dont naissent toujours un peu les apologies. Car pourquoi se charger de justifier l'ordre ancestral de la noblesse s'il n'a rien à craindre? son plaidoyer pro domo, Vauvenargues, homme de demain malgré qu'il en ait, est incapable de l'articuler logiquement. Davantage, un an à peine avant de mourir, il se qualifie dans ses dernières lettres à son meilleur ami, comme s'il craignait de demeurer en reste sur la marche des idées, de "philosophe". ${ }^{23} \mathrm{~A}$ la perspective d'être laissé pour compte Vauvenargues répugne en effet dans sa chair et dans son âme. Et puis sont-ce principalement les bourgeois, ainsi qu'il semble vouloir nous le faire croire, qui se dérobent à l'activité de la nation? À sa survivance économique? Sont-ils les plus coupables alors que c'est précisément chez eux que se font entendre les appels à la réforme? Quand même leur idéal laisserait sérieusement à désirer, quand il se réduirait à l'expression sublimée de l'esprit d'intérêt, de l'envie sordide des titres et des privilèges, ils prennent historiquement place comme les vrais moteurs de l'expansion nationale et les instigateurs archétypaux de la dynamique révolutionnaire. Et pourtant, peu importe, au fond, que Vauvenargues se soit un instant trompé (s'il est vrai qu'il s'est trompé) de cible. Ce qui compte, ce qui tire à conséquence, c'est qu'il sonne le tocsin pour signaler que quelque chose est pourri au royaume de France et que le pays ne s'affranchira pas impunément de sa pourriture. Il n'en fallait pas tant pour monter les esprits et ouvrir la voie de la révolte.

Ainsi s'explique que les moralistes de la première moitié du siècle aient, souvent à leur corps défendant, concouru à poser les jalons d'un bouleversement qu'ils ne voulaient pas. Emportés tour à tour par le bouillonnement des idées, par la perspective d'un projet de société qui, sans être brutalisant, faisait rêver de justice et de bonheur, ou encore par la certitude de vivre une des heures les plus extraordinaires de l'histoire de l'humanité, ils ne se prenaient pas que les témoins objectifs d'un âge, ils se voulaient aussi ses prophètes et ses maîtres à penser. Chacun à sa manière, toujours, mais chacun selon une ligne de force qui l'arrachait irrésistiblement, qu'il le désirât ou non, à un passé à jamais révolu. Mais ce faisant, ils culbutèrent dans la politique et 
découvraient pour la première fois que la connaissance de l'homme dépassait infiniment celle de sa nature. Tournant décisif dans l'évolution de la réflexion moraliste (et morale), qui allait jeter les écrivainsrelayeurs comme Sénac de Meilhan, Chamfort et Rivarol au plus fort de la mêlée révolutionnaire et contre-révolutionnaire.

Parents par la pensée, voltairiens par l'esprit (sinon par le coeur), amis même pour l'estime où ils se tiennent mutuellement, ces derniers n'en sont pas moins en effet à eux seuls, à travers leurs divergences, leurs espoirs ou leurs cynismes, comme le microcosme de 1789. A tel point que, encore aujourd'hui, leurs couleurs politiques respectives paraissent être les seuls critères valables pour juger du mérite de leurs oeuvres! Ne voit-on pas, par exemple, la critique de droite se réclamer religieusement de Rivarol et celle de gauche le vouer au diable? Comme si l'une en faisait son bréviaire et l'autre son Index! Car quel adversaire redoutable pour les partisans d'un ordre nouveau que l'auteur du Journal politique et national et du Petit Dictionnaire des grands hommes de la Révolution par un citoyen actif, ci-devant rien! D'une justesse pénétrante dans ses aperçus sur l'histoire et l'évolution du jour ${ }^{24}$ (peutêtre même la plus pénétrante de toute la pensée contre-révolutionnaire) et d'un tour d'esprit particulièrement meurtrier dans sa raillerie, il rappelle, dans une plume étourdissante et malgré le dégoût où le met l'insane arrogance du noble avec sa décrépitude morale, tout ce que la France doit de reconnaissance filiale à l'Ancien Régime.

Dans le camp opposé, Chamfort, le frère de coeur et d'intelligence, exaltant dans l'euphorie la Révolution par laquelle se cristallise enfin à ses yeux, après des siècles d'attente et de misère, la maturation sociale et politique de l'humanité, Chamfort dispense aux Mirabeau et Sieyès, à tous les défenseurs des idées nouvelles qui viennent puiser sans vergogne aux sources de ses extraordinaires dons d'écrivain, les formules les plus frappantes, les traits les plus percutants de l'éloquence révolutionnaire. Lorsque le 15 décembre 1792, par exemple, Cambon termine son discours sur le: "guerre aux châteaux, paix aux chaumières", il reprend à son compte le slogan de Chamfort. Et c'est Chamfort encore qui souffle à l'oreille de Sieyès le titre de sa fameuse brochure: "Qu'est-ce que le Tiers-Etat? Tout -- Qu'a-t-il? Rien." Nombreux même sont ceux qui, tel Lauraguais, réprouvent, comme évoquant l'aumône, le simple "quelque chose" qu'y ajoute Sieyès. ${ }^{25} \mathrm{C}^{\prime}$ est l'abandon des principes révolutionnaires que représente pour lui la crise du printemps 93, c'est le refus, logeant au plus profond de sa dignité, de vivre et de mourir autrement qu'en homme libre, bref, c'est l'idéal perdu, le beau rêve éclaté qui finira par avoir raison de Chamfort, de 
cette manière d'André Malraux du XVIIIe siècle dont la légende veut encore qu'il soit entré des premiers à la Bastille.

Sur un autre front, du côté de Rivarol et de ceux qui défendent la royauté, Sénac de Meilhan, qui depuis toujours a abjuré le doute et le désespoir, de crainte peut-être de finir en tragique héros de l'absurde comme, plus tard, Chamfort, continue à prendre ses rêves pour des réalités et à rassurer son auditoire. "De nos jours, écrit-il en 1787 dans ses Considerations sur l'esprit et les moeurs, la Puissance des Souverains est assise sur des bases inébranlables. Des armées nombreuses s'opposent aux troubles intérieurs, ainsi qu'aux invasions promptes." 26 Qui se risquerait, après avoir affiché un tel manque de discernement -- j'allais dire "une telle naïveté", mais elle était d'époque dans la noblesse qui se refusait à rien voir qui la dérangeât --, qui se risquerait à poursuivre sur ce même terrain? Non, chat échaudé ne craint pas toujours l'eau froide, et Sénac, trois ans plus tard, alors que les ébranlements ne font encore que commencer, publie sous un titre ronflant qui mettait comme un terme politique au sens des événements de 89 un Des principes et des causes de la Révolution! Pourtant, sans y songer, un peu à la façon des moralistes (et philosphes) qui l'avaient préparée idéologiquement sans la vouloir, il fournit à la Révolution montante des armes d'autant plus insidieuses qu'elles sont fondées sur un principe de moralité absolue et dans les formes, non d'un a priori utopiste, mais de la contingence empirique. Dans son chapitre des Considérations sur les républiques et les monarchies, par exemple, chapitre où il s'emploie à peser le pour et le contre des deux systèmes de gouvernement, il écrit: "Dans les Républiques, chacun a le développement et l'exercice de ses facultés. Dans les Monarchies, l'homme qui a de l'énergie, n'a souvent d'autres ressources que les plaisirs". ${ }^{27}$ Et ailleurs: "Dans les Républiques, on dépend des lois plus que des personnes. La sécurité où l'on vit rend les ménagements inutiles, et il y a plus d'égalité parmi les citoyens et plus de liberté dans les esprits". ${ }^{28}$ "Dans les Monarchies, la crainte du ridicule doit dominer les esprits et produire un asservissement à la mode, qui affoiblit et qui corrompt" ${ }^{29}$ Paradoxalement, Sénac, le regard tourné vers l'avenir malgré qu'il en ait, invite les Français à prendre des options sur une nouvelle société et sur un homme nouveau. Davantage: treize ans plus tard, après avoir tout perdu par la faute de la Révolution, il fait une croix idéologique sur les bienfaits dont la Royauté l'avait comblé, et met dans la bouche du président de Longueil, sous les traits duquel il se peint dans l'Emigré: "La révolution de la France, unique dans son espèce, a donné aux esprits une commotion violente, qui leur a fait parcourir en tous sens les sentiers de l'économie politique et de la législation. Les Français, charmés de 
leur indépendance, se sont livrés aux plus téméraires conceptions; ils ont détruit, mais ils ont en même temps creusé, porté la lumière dans les routes les plus obscures, et ils en ont ouvert de nouvelles et forcé les barrières élevées par le préjugé. Un jour viendra où dans le calme on examinera ces nombreuses discussions enfantées au milieu du tumulte et de l'effervescence de l'esprit de parti, et l'on fera paisiblement un choix éclairé de résultats utiles à l'humanité". ${ }^{30}$ Acte de foi dans le progrès politique et social s'il en est, car ce "jour", Sénac sait très bien qu'il ne le verra jamais, comme il sait que l'émigré qu'il est depuis 1791, ne foulera plus de sa vie le sol de France.

Que conclure? Je me contenterai de faire trois remarques. La première est que, contrairement à ce que l'on dit encore trop souvent, les moralistes des Lumières sont frères (voire, à l'occasion, frères aînés) des philosophes; ils exaltent un idéal d'être et, surtout à partir de 1750, d'ordre politique qui balise le tracé de la route révolutionnaire. La seconde, c'est que, par leur vision (même chez Rivarol) ${ }^{31} \mathrm{~d}^{\prime}$ une humanité en devenir, ils apparaissent comme les tenants originaires du déterminisme historique; d'où le passage de la nature humaine comme objet de réflexion à l'homme social comme projet politique..$^{32}$ Enfin, et ce sera ma troisième remarque, les moralistes des Lumières nous apprennent, presque toujours à leurs dépens, qu'il ne saurait y avoir de parole gratuite dans quelque commerce des hommes que ce soit; qu'on ne mesure quasi jamais la portée de ce qu'on dit et que, l'on a beau se proposer d'écrire pour quelques initiés, on finit toujours par se faire entendre de la multitude.

HENRI MYDLARSKI

University of Calgary

NOTES

1. Vauvenargues, Oeuvres et Oeuvres posthumes et oeuvres inédites, éd. D.-L. Gilbert (Paris, 1857), I, 374.

2. Vauvenargues, op. cit., I, 374.

3. Richard $M$. Chadbourne écrit à ce propos que les Français ont un véritable "genius for criticism and for la littérature des moralistes". Il ajoute même, mais là la remarque demanderait à être approfondie et justifiée selon des critères génériques rigoureux, que ce "genius ... may have worked to overshadow what is in fact their [of the French] real versatility as essayists", "A Puzzling Literary Genre: Comparative Views of the Essay", Comparative Literature Studies, XX, No. 2 (Eté 1983), 147. 
4. André Siegfried, L'Ame des peuples (Paris: Hachette, 1950), p. 57.

5. Vauvenargues, op. cit., I, 400.

6. Henri Baudrillart, "Vauvenargues", La Liberté de penser. Revue philosophique et littéraire, IV, No. 19 (Juin 1849), 30. Article reproduit dans Etudes de philosophie morale et d'économie politique du même auteur, Tome I, Paris: Guillaumin et Cie, 1858.

7. Henri Baudrillart, op. cit., p. 30 .

8. Tel est le titre du premier volume de la seconde "trilogie" de Paul Hazard: La pensée européenne au XVIIIe siècle; de Montesquieu à Lessing.

9. Henri Baudrillart, op. cit., p. 21.

10. Dominique Julia écrit par exemple: “Christianiser les campagnes, c'est $\mathrm{d}$ 'abord façonner des comportements par une discipline qui rend les corps et les coeurs dociles. Il s'agit, pour l'éducation des conduites, de civiliser une animalité", "la plupart des paysans contractant ordinairement par le commerce qu'ils ont avec les bêtes une nature moyenne entre leur façon de vivre et celle de l'homme politique", "Discipline ecclésiastique et culture paysanne aux XVIIle et XVIIIle siècles", dans La Religion populaire (Paris: Eidtion du C.N.R.S., 1979), pp. 208-209. On pourrait aller plus loin et dire que la discipline ecclésiastique ne saurait pas s'appliquer qu'aux seuls paysans. Et on rappellerait à ce propos la fameuse injonction que fait Pascal de "s'abêtir", injonction qui ne s'adresse évidemment pas aux analphabêtes ni aux "abrutis", au sens littéral du mot.

11. Vauvenargues, op. cit., I, 161.

12. [François Vincent Toussaint], Les Moeurs (S.1., n.d. 1748), p. 66-67.

13. Duclos, Oeuvres complètes, éd. M. Augen (Paris: Janet et Cotelle, 1820-1821), I, 9.

14. Duclos, op. cit., I, 192.

15. [François Vincent Toussaint], op. cit., p. 105.

16. Jacques Vier, "La prose d'idées au XIXe siècle, "dans Histoire des Littératures III (Paris: "La Pléiade", 1958), p. 1209. Encore que fait à l'occasion de Renan, cette remarque se prête à merveille à l'étude des moralistes des Lumières.

17. cf. Anne-Marie Desfougères, "Des moralistes à la psychanalyse", in Destins et enjeux du XVIle siècle (Paris: P.V.F., 1985), p. 251.

18. Duclos, op. cit., I, 21.

19. Vauvenargues, op. cit., II, 68.

20. Vauvenargues, op. cit., I, 429.

21. Vauvenargues, op. cit., I, 71. En marge de son fameux exemplaire de la 1ère édition de Vauvenargues, exemplaire qui se trouve à la bibliothèque Méjanes d'Aix, Voltaire a noté: "Bien et neuf". Ce qui prouve bien, s'il était encore besoin de le prouver, que Voltaire n'a jamais cherché à renverser les institutions les plus susceptibles d'être remises en question et qu'il était bien loin d'être un révolutionnaire. D'où, évidemment, on ne saurait conclure que les causes de la Révolution ne se rencontrent pas aussi en lui, à l'image duquel tout le siècle semble s'identifier et se créer chaque jour davantage. 
22. Vauvenargues, op. cit., I, 166.

23. Voir Vauvenargues, op. cit., II, 295 et 296.

24. Rivarol est l'un des tout premiers, peut-être même le premier, à avoir, par exemple, compris que la Révolution française constituait le modèle même de la Révolution nationale bourgeoise. Voici en partie ce qu'il écrit à ce propos: "Ce ne sont ni les impôts, ni les lettres de cachet, ni tous les autres abus de l'autorité; ce ne sont point les vexations des intendants, et les longueurs ruineuses de la justice, qui ont le plus irrité la nation; c'est le préjugé de la noblesse, pour lequel elle a manifesté plus de haine; ce qui prouve évidemment que ce sont les bourgeois, les gens de lettres, les gens de finances, et enfin tous ceux qui jalousaient la noblesse, qui ont soulevé contre elle le petit peuple dans les villes, et les paysans dans les campagnes". Oeuvres complètes, 5 vol. (Paris: Léopold Collin, 1808), IV, 101. Rivarol se plaisait même à dire que les nobles de son temps n'étaient plus que "les mânes de leurs ancêtres" (IV, 338). Par là il rejoignait Vauvenarges qui s'était, en vain, essayé de les remettre sur la voie de l'honneur et du mérite avant qu'il ne fût trop tard.

25. Voir Julien Teppe, Chamfort - sa vie, son oeuvre, sa pensée (Paris: Edition Pierre Clairac, 1950), p. 49.

26. Sénac de Meilhan, Considérations sur l'esprit et les moeurs (Paris: E. Sansot et Cie., 1905), p. 78.

27. Sénac de Meilhand, op. cit., p. 90.

28. Sénac de Meilhan, op. cit., p. 91.

29. Sénac de Meilhan, op. cit., pp. 90-91.

30. Sénac de Meilhan, L'Emigré in Romanciers du XVIIIe siècle, II, éd. R. Etiemble (Paris: "La Pléiade", 1965), pp. 1751-1752.

31. Rivarol écrit par exemple: "Montagne, Charron, Bayle, la Motte-le-Vayer et autres, parlèrent hardiment de tout; mais ces semences de liberté se perdirent sur un terrain mal préparé", op. cit., II, 120. Par cette remarque Rivarol atteste qu'il existe au moins une histoire des mentalités, histoire à laquelle, d'ailleurs, les Français ne semblent véritablement s'intéresser que depuis quelques années.

32. C'est encore Rivarol qui écrit: "Enfin l'homme de la nature, ce n'est pas l'homme solitaire, mais l'homme social", op. cit., I, 358. Et cette constatation, Vauvenargues aurait été le premier à y souscrire. 